\title{
Health care inequality under different medical insurance schemes in a socioeconomically underdeveloped region of China: a propensity score matching analysis
}

Wei Xian ${ }^{1,2}$, Xueying $\mathrm{Xu}^{1}$, Junling $\mathrm{Li}^{1}$, Jinbin Sun ${ }^{1}$, Hezi Fu${ }^{3}$, Shaoning $\mathrm{Wu}^{1}$ and Hongbo Liu ${ }^{1 *}$ (D)

\begin{abstract}
Background: Since economic inequality is often accompanied by health inequalities, health care inequalities are increasingly becoming a hot issue on a global scale. As a developing country, China is still facing the same problems as other countries in the world. Especially in underdeveloped regions, owing to the relatively backward economy, health care inequality may be more serious. The objective of this study was to explore health care inequality in a socioeconomically underdeveloped city, thus providing a certain theoretical basis for further development and reform of the medical insurance schemes.
\end{abstract}

Methods: We mainly extracted relevant insurance information of 628,952 insured enrollees, as well as consumption of outpatient visit and hospitalization. The propensity score matching had been used to estimate different urban medical insurance schemes effect on healthcare utilization, the choice of hospital types and healthcare cost.

Results: Insured enrollees spent most hospitalization expenses in tertiary-level hospitals, which had lowest hospitalization compensation ratios. Healthcare utilization and cost vary significantly by different insurance schemes. Urban employees had significantly higher outpatient visit rates in all hospital types than urban residents. Urban employees preferred to receive hospitalization treatment in tertiary-level hospitals, while those who receive hospitalization treatment in first-level hospitals are more likely to be enrolled in Urban Residents Basic Medical Insurance. Hospitalization expenses and hospitalization compensation ratios of urban employees were also significantly higher than urban residents in all hospital types.

Conclusions: Health care inequality is mainly reflected in the imbalance between hospitalization expenses and hospitalization compensation ratios, as well as inequalities under different medical insurance schemes in healthcare utilization, the choice of hospital types and healthcare cost in socioeconomically underdeveloped regions of China. We should conduct a targeted medical insurance reform for the socioeconomically underdeveloped regions, rather than applying templates of ordinary regions. Further efforts are needed in the future to provide equal health care for every patient.

Keywords: Health care inequality, Healthcare utilization, Healthcare cost, Propensity score, Propensity score matching

\footnotetext{
* Correspondence: hbliu@cmu.edu.cn

'School of Public Health, China Medical University, Shenyang, People's

Republic of China

Full list of author information is available at the end of the article
}

(c) The Author(s). 2019 Open Access This article is distributed under the terms of the Creative Commons Attribution 4.0 International License (http://creativecommons.org/licenses/by/4.0/), which permits unrestricted use, distribution, and reproduction in any medium, provided you give appropriate credit to the original author(s) and the source, provide a link to the Creative Commons license, and indicate if changes were made. The Creative Commons Public Domain Dedication waiver (http://creativecommons.org/publicdomain/zero/1.0/) applies to the data made available in this article, unless otherwise stated. 


\section{Background}

Since economic inequality is often accompanied by health inequalities, health care inequalities are increasingly becoming a hot issue on a global scale [1-4]. As people pay attention to their own health, health care inequalities are getting more and more attention from the public. As a developing country with fast economic growth, China also faces the same problems as other countries in the world. According to the World Health Report in 2000, China's overall health system performance ranked 144 out of 199 countries and the ranking of equity in health financing was third from last in the world [5]. There are two main reasons for this phenomenon. One of them is insufficient coverage of medical insurance [6], and the other is health care inequality. In the past 20 years, China has achieved a series of health reforms $[7,8]$. It included further coverage of Urban Employees Basic Medical Insurance (UEBMI) and Urban Residents Basic Medical Insurance (URBMI). UEBMI is a social insurance system established in 1998 to compensate urban employees for economic losses due to disease risks. However, the elderly, children, students, and urban non-employed residents are not included in this scheme. Therefore, in order to reduce the medical expenses brought by the growing health needs of residents, URBMI has been implemented since 2007. The implementation of URBMI indeed makes up for the health care of some groups and improves health care inequalities. The coverage rate of medical insurance in China has exceeded 95\% [9, 10]. But does this fastdeveloping insurance system mask undetectable health care inequalities and fail to achieve real health care inequalities? With the coverage of universal medical insurance in China, health care inequality has become more important and needs to be taken seriously. Health care inequality is mainly reflected in the imbalance between hospitalization expenses and hospitalization reimbursement, as well as inequalities under different medical insurance schemes in the use of medical resources and the choice of hospital types.

In today's China, citizens can directly go to any level of hospital, but they are still more willing to choose high-level hospitals for treatment [11]. In order to alleviate the pressure on high-level hospitals, China has implemented grading diagnosis and treatment system, including a two-way referral system, to leave some patients with slight illness in low-level hospitals. However, it still cannot completely eliminate the phenomenon of "inverted pyramids" in China. In some cities, there are still many patients gathered in high-level hospitals to see a doctor [12]. Patients believe that they can get relatively better treatment in high-level hospitals [11]. In addition to focusing on better treatment, patients are also very concerned about medical expenses. So does the phenomenon of "inverted pyramids" exist in socioeconomically underdeveloped regions? Does the hospital with the most hospitalization expenses have the most hospitalization reimbursement? These issues are worthy exploring further.

Although the use of health care should be distributed equitably based on people's needs rather than ability to pay [13], the current use of health care is unequal. Healthcare utilization is more beneficial to the rich [14]. The rich enjoy the relatively high-level healthcare utilization, while the poor have to choose lower-level services or even no money to get health care $[15,16]$. So there is such a saying that it is difficult and expensive to see a doctor. Underdeveloped regions are relatively backward in economy. In these regions, the difficulty and high expense for getting medical treatment may be more serious. Therefore, the use of medical resources and the choice of hospital types may be different from those in ordinary regions and require more attention. Since different insurance schemes have an impact on healthcare utilization and costs $[17,18]$, it is worth considering whether different insurance schemes will have a greater impact on healthcare utilization, the choice of hospitals and healthcare cost in socioeconomically underdeveloped regions. Earlier studies focused more on ordinary regions and did not use propensity scores to reduce the effects of confounding factors [19-22], which had increased bias in the research.

Medical inequality is easily hidden by economic development, so our aim was to explore whether there is an imbalance between hospitalization expenses and hospitalization reimbursement, and whether there are inequalities under different medical insurance schemes in healthcare utilization, the choice of hospital types and healthcare cost in a socioeconomically underdeveloped city of China. Our paper contributes to a certain theoretical basis for policy makers to pay more attention to health inequalities, thus eliminating the greater hidden dangers in the future.

\section{Methods \\ Study setting}

Fuxin city is the typical socioeconomically underdeveloped city in China. The economy of Fuxin city is relatively backward compared to ordinary cities in China [23]. The urban population is 1.04 million. About $70 \%$ of them participate in UEBMI or URBMI.

\section{Data source}

The data in this study were derived from Fuxin Social Insurance Bureau. Fuxin Social Insurance Bureau collected all medical insurance data annually from all insured enrollees in Fuxin city. Data related to insured information will be updated at any time. We mainly extracted relevant insurance information of insured 
enrollees in 2015, as well as consumption of outpatient visit and hospitalization.

UEBMI enrollees were aged 17 and older, while URBMI enrollees included many infants and children. However, infants and children were susceptible to various diseases, often accompanied by greater medical expenses compared to people of other ages. We excluded this small proportion of insured enrollees to prevent differences in medical expenses and age differences after matching. Therefore, insured enrollees aged 17 and older became the research subjects of this study.

The demographic characteristic and medical expenses of insured enrollees were extracted. The demographic characteristic mainly included gender, date of birth, workplaces (enterprises, individual, state-owned workplaces and other workplaces), and wages (low wages, medium wages, high wages). We defined ages into three groups: $17-45,45-60$, and 60-. According to average social wages of employees in Fuxin city, the wages are divided into three levels: low, medium and high. In addition, we collected the dates of visit, hospital types, out-of-pocket expenses, insurance expenses, and total expenses related to outpatient visit and hospitalization.

\section{Statistical methods}

The propensity score was first proposed by Rosenbaum and Rubin in the 1980s [24]. Propensity score matching (PSM) is to make the selected two groups comparable in terms of potential confounding factors, in order to balance variables and reduce bias. In the first step of PSM analysis, a series of demographic variables which might impact on healthcare utilization, hospital types and healthcare cost were considered to calculate the propensity score. The selected variables were based on data availability and literature review, including gender, age, workplaces, and wages. We performed multivariate logistic regression to calculate the propensity score. In the second step of PSM analysis, Greedy matching techniques, proposed by Parsons [25], was chosen to perform one-to-one matching. After matching, the quality of the match was verified by calculating standardized bias and conducting a significance test. Standardized bias are calculated by the formula,

$$
d=\frac{100 *\left(p_{1-} p_{2}\right)}{\sqrt{\frac{p_{1}\left(1-p_{1}\right)+p_{2}\left(1-p_{2}\right)}{2}}}
$$

where $p_{1}$ represents the group of URBMI enrollees and $p_{2}$ represents the group of UEBMI enrollees. When standardized bias is less than 0.1 , the covariates between the two groups are balanced and there is no difference between them.

The chi-square test was adopted to estimate different medical insurance schemes effect on healthcare utilization and the choice of hospital types. Hospital types are divided into three levels, namely the first-level hospital, the secondary-level hospital and the tertiary-level hospital. Firstlevel hospitals correspond to low-level hospitals, and tertiary hospitals correspond to high-level hospitals. Non-parametric test was used to assess the impact of different medical insurance schemes on healthcare cost. We mainly used hospitalization expenses and hospitalization compensation ratio as a measure of healthcare costs. Hospitalization compensation ratio refers to the ratio of the amount reimbursed by medical insurance to the total hospitalization expenses.

All indicators were calculated using SAS software, version 9.4. None of the funders had any role in the design of the study and collection, analysis, and interpretation of data and in writing the manuscript.

\section{Results}

Demographic characteristics of all insurance enrollees There were 628,952 people participating in medical insurance, including 549,603 UEBMI enrollees and 79,349 URBMI enrollees. Table 1 reported the summary statistics of key variables. The results indicated the trends of hospitalization expenses and hospitalization compensation ratios in different hospital types were completely opposite. Insured enrollees spent most hospitalization expenses in tertiary-level hospitals, which had lowest hospitalization compensation ratios. However, first-level hospitals with highest hospitalization compensation ratios had the lowest hospitalization expenses. This phenomenon had appeared in both insurance schemes. It is not difficult for us to find an unreasonable phenomenon. That is, there has been an imbalance between hospitalization expenses and hospitalization compensation ratios in a socioeconomically underdeveloped region.

\section{Match evaluation}

In order to better compare different insurance schemes, it is necessary to balance basic demographic variables. Table 2 showed the multivariate logistic regression results for propensity scores. Age, gender, workplaces, and wages between UEBMI enrollees and URBMI enrollees were statistically significant. A one-to-one match was made based on the generated propensity scores. The final sample size for analysis was 36,031 UEBMI enrollees and 36,031 URBMI enrollees.

The PSM improved comparability between UEBMI enrollees and URBMI enrollees. Standardized bias for all variables between UEBMI enrollees and URBMI enrollees were lower than $5 \%$ after matching. By matching, all variables were balanced between the two groups. In addition, on the basis of the chi-square test, the difference between UEBMI enrollees and URBMI enrollees was statistically insignificant for all characteristics 
Table 1 Summary statistics of key variables $(N=628,952)$

\begin{tabular}{|c|c|c|c|}
\hline & & $\begin{array}{l}\text { UEBMI enrollees } \\
(N=549,603)\end{array}$ & $\begin{array}{l}\text { URBMI enrollees } \\
(N=79,349)\end{array}$ \\
\hline \multicolumn{4}{|c|}{ Basic demographic variables (number/percent) } \\
\hline \multirow[t]{3}{*}{ Age (years) } & $17-45$ & $180,430(32.83 \%)$ & $23,832(30.03 \%)$ \\
\hline & $45-59$ & $229,889(41.83 \%)$ & $26,501(33.40 \%)$ \\
\hline & $>59$ & $139,284(25.34 \%)$ & $29,016(36.57 \%)$ \\
\hline \multirow[t]{2}{*}{ Gender } & Male & $276,043(50.23 \%)$ & $30,576(38.53 \%)$ \\
\hline & Female & $273,560(49.77 \%)$ & $48,773(61.47 \%)$ \\
\hline \multirow[t]{4}{*}{ Workplaces } & Enterprise & $273,185(49.71 \%)$ & $345(0.43 \%)$ \\
\hline & Individual & $180,548(32.85 \%)$ & $282(0.36 \%)$ \\
\hline & State-owned workplaces & $92,593(16.85 \%)$ & $78,717(99.20 \%)$ \\
\hline & Other workplaces & $3277(0.60 \%)$ & $5(0.01 \%)$ \\
\hline \multirow[t]{3}{*}{ Wages } & Low & $354,049(64.42 \%)$ & $63,658(80.23 \%)$ \\
\hline & Medium & $42,355(7.71 \%)$ & $2958(3.73 \%)$ \\
\hline & High & $153,199(27.87 \%)$ & $12,733(16.05 \%)$ \\
\hline \multicolumn{4}{|l|}{ Outpatient visit (\%) } \\
\hline \multicolumn{2}{|c|}{ Any outpatient visit in the past year } & 17.47 & 1.14 \\
\hline \multirow[t]{3}{*}{ Hospital types } & First-level hospital & 9.66 & 0.10 \\
\hline & Secondary-level hospital & 2.12 & 0.94 \\
\hline & Tertiary-level hospital & 8.41 & 0.14 \\
\hline \multicolumn{4}{|l|}{ Hospitalization (\%) } \\
\hline \multicolumn{2}{|c|}{ Any hospitalization in the past year } & 14.09 & 12.20 \\
\hline \multirow[t]{3}{*}{ Hospital types } & First-level hospital & 1.73 & 1.31 \\
\hline & Secondary-level hospital & 4.92 & 4.87 \\
\hline & Tertiary-level hospital & 8.72 & 7.12 \\
\hline \multicolumn{4}{|c|}{ Hospitalization expenses (median/ lower quartile upper quartile, RMB) } \\
\hline \multirow[t]{3}{*}{ Hospital types } & First-level hospital & $3075.10(2183.99 \sim 4934.57)$ & 2990.65 (1919.77 4610.90) \\
\hline & Secondary-level hospital & $4611.98(2942.49 \sim 7294.23)$ & $4002.23(2467.00 \sim 6689.57)$ \\
\hline & Tertiary-level hospital & $6085.53(3979.50 \sim 10,396.24)$ & $5503.85(3491.24 \sim 9630.11)$ \\
\hline \multicolumn{4}{|c|}{ Hospitalization compensation ratios (median/lower quartile upper quartile, \%) } \\
\hline \multirow[t]{3}{*}{ Hospital types } & First-level hospital & 77.81 (73.76 82.35) & $69.81(63.20 \sim 81.81)$ \\
\hline & Secondary-level hospital & $76.36(71.50 \sim 81.31)$ & $70.02(62.03 \sim 81.25)$ \\
\hline & Tertiary-level hospital & 67.81 (62.86 72.54) & 58.74 (52.24 72.15) \\
\hline
\end{tabular}

$(p>0.05)$. Detailed PSM evaluation results were shown in Tables 3.

\section{The effect of medical insurance schemes on healthcare utilization}

Table 4 presented outpatient visit rates and hospitalization rates for different hospital types under different medical insurance schemes. We can see that any outpatient visit in the past year of UEBMI enrollees and URBMI enrollees were statistically different. Among all hospital types, UEBMI enrollees had significantly higher outpatient visit rates than URBMI enrollees. Insured enrollees in the UEBMI scheme increased the use of outpatient services. There was also a statistical difference in any hospitalization in the past year between the two groups. However, hospitalizations of both groups were only statistically different in first-level and tertiary-level hospitals. UEBMI enrollees had significantly higher hospitalization rate in tertiary-level hospitals, while hospitalization rate of URBMI enrollees was higher in first-level hospitals. Compared with the possibility of URBMI enrollees being hospitalized, UEBMI enrollees were more likely to receive treatment in tertiary-level hospitals rather than in first-level hospitals.

The effect of medical insurance schemes on healthcare cost Figures 1 and 2 showed the distribution of hospitalization expenses and hospitalization compensation ratios for different hospital types under different insurance schemes. 
Table 2 Multivariate logistic regression results for propensity scores

\begin{tabular}{|c|c|c|c|}
\hline & Estimate & Standard Error & Odds Ratio (95\% Confidence Interval) \\
\hline \multicolumn{4}{|l|}{ Age (years) } \\
\hline $17-45$ & Reference & & \\
\hline $45-59$ & -0.404 & 0.015 & $0.668(0.648,0.688)^{* *}$ \\
\hline$>59$ & 1.103 & 0.017 & $3.014(2.913,3.118)^{* *}$ \\
\hline \multicolumn{4}{|l|}{ Gender } \\
\hline Male & Reference & & \\
\hline Female & -0.608 & 0.012 & $0.545(0.532,0.558)^{* *}$ \\
\hline \multicolumn{4}{|l|}{ Workplaces } \\
\hline Enterprise & 1.066 & 0.452 & $2.905(1.198,7.043)^{*}$ \\
\hline Individual & 1.887 & 0.453 & $6.597(2.717,16.022)^{* *}$ \\
\hline State-owned workplaces & -6.350 & 0.449 & $0.002(<0.001,0.004)^{* *}$ \\
\hline Other workplaces & Reference & & \\
\hline \multicolumn{4}{|l|}{ Wages } \\
\hline Low & Reference & & \\
\hline Medium & 2.352 & 0.026 & $10.504(9.991,11.043)^{* *}$ \\
\hline High & 3.256 & 0.016 & $25.946(25.126,26.794)^{* *}$ \\
\hline
\end{tabular}

No matter what hospital types, UEBMI enrollees and URBMI enrollees had statistically significant differences in hospitalization expenses and hospitalization compensation ratios. Furthermore, hospitalization expenses and hospitalization compensation ratios of UEBMI enrollees were both higher than those of URBMI enrollees in all hospital types.

\section{Discussion}

Providing equal health care to every patient is the goal of all countries around the world. China is also making unremitting efforts to this end. In the past, China has made some progress in providing affordable and equitable basic medical services to the population, which has surpassed many developing countries. However, health care inequalities enjoyed by each subgroup of insurance enrollees still exist. This paper indicated the imbalance between hospitalization expenses and hospitalization reimbursement, and systematically estimated the impact of different medical insurance schemes on health care inequality in healthcare utilization, the choice of hospital types and healthcare cost in a socioeconomically underdeveloped city.

In a socioeconomically underdeveloped city, the phenomenon of "inverted pyramids" still exists. In the inherent misconceptions of many Chinese people, the need for hospitalization indicates a very serious disease, and high-level hospitals can get good treatment. At this

Table 3 Comparison of sample characteristics before and after propensity score matching (PSM)

\begin{tabular}{|c|c|c|c|c|c|c|c|c|}
\hline & \multicolumn{4}{|l|}{ Before PSM } & \multicolumn{4}{|l|}{ After PSM } \\
\hline & UEBMI (\%) & URBMI (\%) & Standardized Bias (\%) & $P$ value & UEBMI (\%) & URBMI (\%) & Standardized Bias (\%) & $P$ value \\
\hline \multicolumn{9}{|l|}{ Age (years) } \\
\hline $45-59$ & 41.83 & 33.40 & -17.47 & $<0.001$ & 34.12 & 34.02 & -0.21 & 0.777 \\
\hline$>59$ & 25.34 & 36.57 & 24.47 & $<0.001$ & 39.81 & 39.89 & 0.16 & 0.831 \\
\hline Female & 49.77 & 61.47 & 23.71 & $<0.001$ & 60.95 & 60.92 & -0.06 & 0.927 \\
\hline \multicolumn{9}{|l|}{ Workplaces } \\
\hline Enterprise & 49.71 & 0.43 & -138.21 & $<0.001$ & 0.95 & 0.96 & 0.10 & 0.970 \\
\hline Individual & 32.85 & 0.36 & -97.04 & $<0.001$ & 0.79 & 0.78 & -0.11 & 0.966 \\
\hline State-owned workplaces & 16.85 & 99.02 & 302.68 & $<0.001$ & 98.25 & 98.25 & 0 & 1.000 \\
\hline \multicolumn{9}{|l|}{ Wages } \\
\hline Medium & 7.71 & 3.73 & -17.20 & $<0.001$ & 8 & 8.21 & 0.77 & 0.306 \\
\hline High & 27.87 & 16.05 & -28.85 & $<0.001$ & 35.55 & 35.34 & -0.44 & 0.559 \\
\hline
\end{tabular}


Table 4 Effect of different medical insurance schemes on healthcare utilization

\begin{tabular}{|c|c|c|c|c|c|}
\hline & & UEBMI (\%) & URBMI (\%) & $x^{2}$ statistics & $P$ value \\
\hline \multicolumn{6}{|l|}{ Outpatient visit } \\
\hline \multicolumn{2}{|c|}{ Any outpatient visit in the past year } & 31.15 & 1.17 & $11,950.495$ & $<0.001$ \\
\hline \multirow[t]{3}{*}{ Hospital types } & First-level hospital & 13.62 & 0.12 & 5125.879 & $<0.001$ \\
\hline & Secondary-level hospital & 4.25 & 0.93 & 785.221 & $<0.001$ \\
\hline & Tertiary-level hospital & 18.85 & 0.15 & 7327.862 & $<0.001$ \\
\hline \multicolumn{6}{|l|}{ Hospitalization } \\
\hline \multicolumn{2}{|c|}{ Any hospitalization in the past year } & 14.95 & 13.36 & 37.105 & $<0.001$ \\
\hline \multirow[t]{3}{*}{ Hospital types } & First-level hospital & 1.26 & 1.51 & 8.422 & 0.004 \\
\hline & Secondary-level hospital & 4.52 & 4.73 & 1.865 & 0.172 \\
\hline & Tertiary-level hospital & 10.09 & 8.37 & 63.467 & $<0.001$ \\
\hline
\end{tabular}

time, people wanted to get the best treatment. Our results showed that first-level hospitals had highest hospitalization compensation ratios. But it was not the first thing they considered. As a result, most hospitalization expenses are still spent in tertiary-level hospitals. This is an abnormal phenomenon that leads to a failure to better allocate and utilize medical resources, thus causing a waste of medical resources. In terms of the choice of hospital types, UEBMI enrollees had higher outpatient visit rates in all hospital types than URBMI enrollees $(p<0.001)$. URBMI enrollees were less likely to use outpatient visit services, probably because URBMI enrollees were unable to get compensation from the insurance scheme and can only use their own money for outpatient visits. The money they have can only guarantee their basic life. So they usually try to minimize the use of outpatient visit services. Our results also indicated that UEBMI enrollees were more willing to receive hospitalization treatment in tertiary-level hospitals $(\mathrm{p}<0.001)$, while those who receive hospitalization treatment in first-level hospitals are more likely to be enrolled in URBMI $(p=0.004)$. We can see that insured enrollees under different insurance schemes have inequalities in the choice of hospital types due to economic inequalities. However, this result is inconsistent with Zhou, et al. [26]. Zhou et al. [26] thought that UEBMI enrollees in Shaanxi Province may be more willing to go to lower-level hospitals for

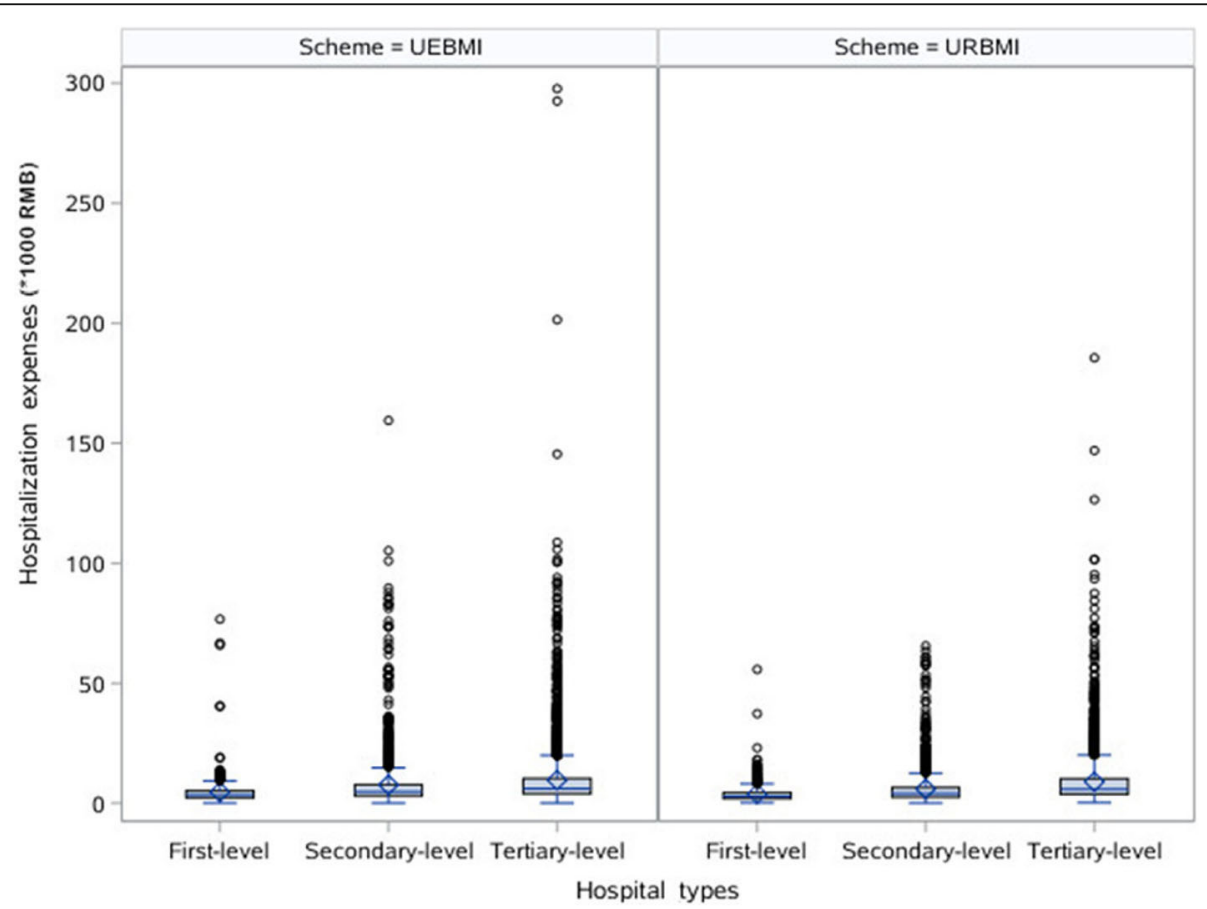

Fig. 1 Hospitalization expenses for different hospital types under different insurance schemes (Comparison of UEBMI enrollees and URBMI enrollees: first-level hospital, $Z=3.931, p<0.001$; secondary-level hospital, $Z=6.824, p<0.001$; tertiary-level hospital, $Z=-2.133, p=0.033$ ) 


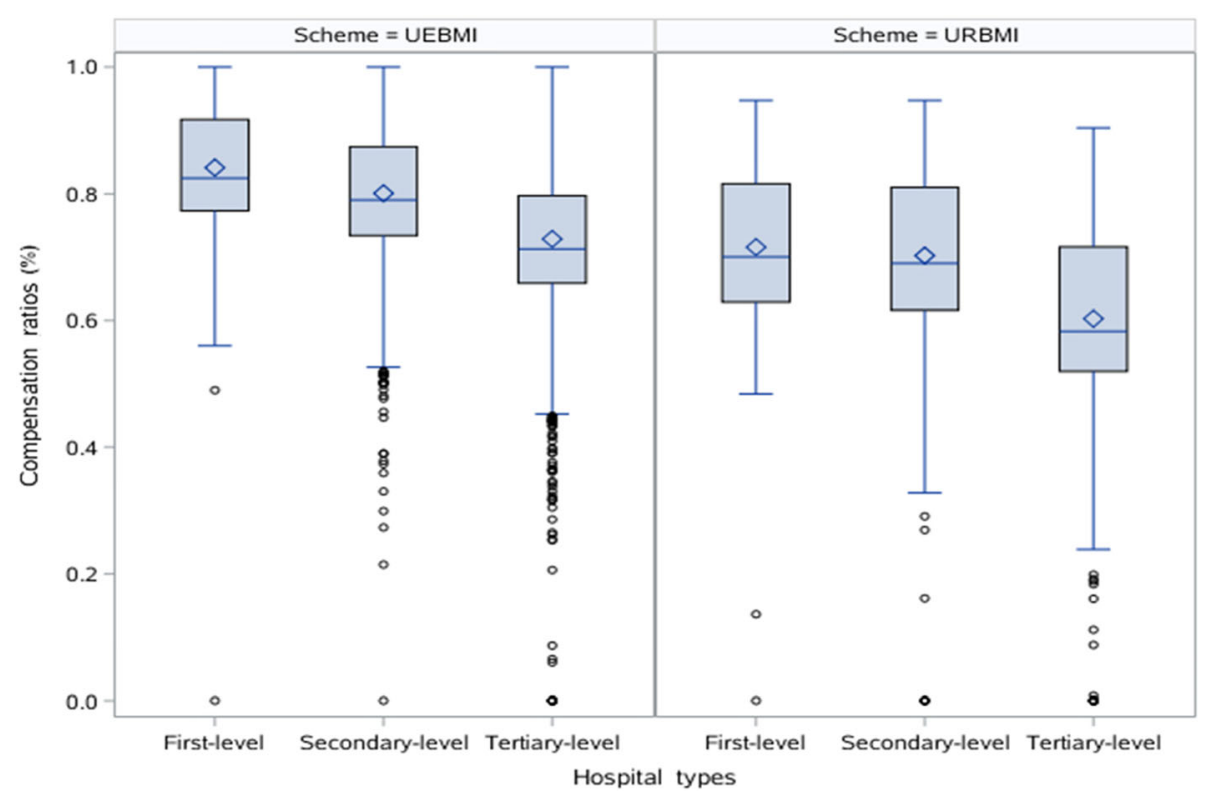

Fig. 2 Hospitalization compensation ratios for different hospital types under different insurance schemes (Comparison of UEBMI enrollees and URBMI enrollees: first-level hospital, $Z=15.366, p<0.001$; secondary-level hospital, $Z=21.266, p<0.001$; tertiary-level hospital, $Z=-36.077, p<0.001$ )

hospitalization. This further explains that the imbalance of hospitalization expenses and hospitalization compensation ratios may be more obvious in a socioeconomically underdeveloped city.

Healthcare utilization varies significantly by different insurance schemes. Insured enrollees in the UEBMI scheme had higher likelihood of healthcare utilization. This may be due to URBMI enrollees have relatively low incomes compared to the UEBMI enrollees. Only when URBMI enrollees have relatively serious illnesses can they go to the hospital for treatment. It showed that the inequalities in the use of medical resources are largely not based on differences in demand but on the economy. Although different insurance schemes target different groups, if there are always huge differences between different insurance schemes, this will bring greater hidden dangers in the future.

Inequalities in healthcare utilization also have left a huge mark on healthcare cost [27]. UEBMI enrollees had higher hospitalization expenses in any hospital type $(p<0.05)$. In addition, hospitalization compensation ratio of UEBMI enrollees was also significantly higher than that of URBMI enrollees $(p<0.001)$. Our findings are consistent with Zhang, et al. [28] in that UEBMI enrollees have the highest compensation ratios and compensation ratios of medical insurance vary significantly by schemes. From this perspective, there is no difference in the hospitalization compensation ratio between socioeconomically underdeveloped regions and ordinary regions. This may be due to the different reimbursement of different urban medical insurance schemes, which also reflects the existence of health care inequality and needs to be valued by government.

Similar to Dou et al. and Yu et al. [29, 30], we conclude that although China has successfully achieved the goal of providing medical insurance for almost the entire population, it faces the challenge of health care inequality. The challenge still continues, and policymakers should now make efforts to reduce health care inequality between subgroups of insurance enrollees. We offer some advices on the current medical insurance policy in socioeconomically underdeveloped regions. The grading diagnosis and treatment system should be further implemented to balance hospitalization expenses and hospitalization compensation ratios. Slight illnesses go to low-level hospitals, and then go to higher-level hospitals. If insurance enrollees participate in this system, the medical insurance reimbursement will increase relatively, and if not, it will fall. We also need to strengthen publicity, improve people's awareness, and make health resources more rationally allocated and utilized. In order to achieve an equality of insurance benefits under different insurance schemes, a large amount of additional public funds is needed. The government can subsidize socioeconomically underdeveloped regions to expand the redistribution of resources.

There are some limitations in our study. By using matching techniques to increase the comparability between different insurance schemes, unobservable (unmatched) characteristics may affect the presented results. During the investigation, we collected data as comprehensively as possible. Completely ideal data is difficult to obtain. In addition, our study did not collect 
information on the existence of chronic diseases, the presence of special occupational diseases, pregnancy status, etc., which may affect the demand for healthcare utilization.

\section{Conclusions}

Healthcare utilization varies significantly by different insurance schemes. Insured enrollees in the UEBMI scheme had higher likelihood of healthcare utilization. Hospitalization expenses and hospitalization compensation ratios of UEBMI enrollees were also significantly higher than those of URBMI enrollees. Health care inequality is mainly reflected in the imbalance between hospitalization expenses and hospitalization compensation ratios, as well as inequalities under different medical insurance schemes in healthcare utilization, the choice of hospital types and healthcare cost in socioeconomically underdeveloped regions of China. We should conduct a targeted medical insurance reform for the socioeconomically underdeveloped regions, rather than applying templates of ordinary regions. Further efforts are needed in the future to provide equal health care for every patient.

\section{Abbreviations \\ PSM: Propensity score matching; UEBMI: Urban Employees Basic Medical Insurance; URBMI: Urban Residents Basic Medical Insurance}

\section{Acknowledgments}

We would like to thank the people who are working in Fuxin Socia Insurance Bureau. Without the help of them, the collected data would not be so complete.

\section{Authors' contributions}

WX conducted the design of study, performed statistical analysis, and wrote the initial manuscript after consultation with the other authors. HL improved the design, revised the manuscript, and approved the final version. XX collected the preliminary data, and helped revise the manuscript. $J$ participated in the design and acquisition of preliminary data. JS collected and sorted the preliminary data. HF and SW participated in the collection of the data. All authors have read and approved the submitted version of the manuscript.

\section{Funding}

This study was supported by the National Natural Science Foundation of Liaoning Province (no.2014021099 to Hongbo Liu) and the Social Sciences Foundation of Liaoning Province (no. L18ATJ001 to Hongbo Liu). None of the funders had any role in the design of the study and collection, analysis, and interpretation of data and in writing the manuscript.

\section{Availability of data and materials}

The datasets used and analyzed during the current study are available from the corresponding author on reasonable request.

\section{Ethics approval and consent to participate}

Our study is a non-interventional study by a retrospective investigation. All procedures were approved by the Ethical Committee Group of China Medical University. In this study, any biomarkers or tissue was not collected, and any individual privacy was not investigated. All data in the study were elicited from insurance records in Fuxin Social Insurance Bureau. The use of the data was approved by them.

\section{Consent for publication}

Not applicable.

\section{Competing interests}

The authors declare that they have no competing interests.

\section{Author details}

${ }^{1}$ School of Public Health, China Medical University, Shenyang, People's Republic of China. ${ }^{2}$ Department of Information Center, The Fourth Affiliated Hospital of China Medical University, Shenyang, People's Republic of China.

${ }^{3}$ Simon Fraser University, Burnaby, Canada.

Received: 7 July 2019 Accepted: 10 October 2019

Published online: 25 October 2019

\section{References}

1. Sommers BD, Mcmurtry CL, Blendon RJ, Benson JM, Sayde JM. Beyond health insurance: remaining disparities in US health Care in the Post-ACA era. Milbank Q. 2017;95(1):43-69. https://doi.org/10.1111/1468-0009.12245.

2. Dickman SL, Himmelstein DU, Woolhandler S. Inequality and the health-care system in the USA. Lancet. 2017;389(10077):1431-41. https://doi.org/10. 1016/S0140-6736(17)30398-7.

3. Fujita M, Sato Y, Nagashima K, Takahashi S, Hata A. Income related inequality of health care access in Japan: a retrospective cohort study. PLOS One. 2016;11(3):e0151690. https://doi.org/10.1371/journal.pone.0151690.

4. Vásquez F, Paraje G, Estay M. Income-related inequality in health and health care utilization in Chile, 2000-2009. Rev Panam Salud Publica. 2013:33(2):981062 p preceding 98.

5. World Health Organization. The world health report 2000-health systems: improving performance. Geneva: WHO; 2000. http://www.who.int/whr/2 000/en/whro0_en.pdf?ua=1

6. Zhu K, Zhang L, Yuan S, Zhang X, Zhang Z. Health financing and integration of urban and rural residents' basic medical insurance systems in China. Int J Equity Health. 2017;16(1):194. https://doi.org/10.1186/s12939-017-0690-z.

7. Xin $\mathrm{H}$. Experiences and lessons from urban health insurance reform in China. Popul Health Manag. 2016;19(4):291-7. https://doi.org/10.1089/pop.2015.0086.

8. Wang C, Rao K, Wu S, Liu Q. Health care in China: improvement, challenges, and reform. Chest. 2013;143(2):524-31. https://doi.org/10.1378/chest.12-1839.

9. National Bureau of Statistics of China. http://data.stats.gov.cn/index.htm.

10. Wang J. Chinese medical service and medical education in urgent need of reform in the context of public welfare-based medical reform. J Biomed Res. 2017;31(3):175-6. https://doi.org/10.7555/JBR.31.20150099.

11. Yip WC, Hsiao WC, Chen W, Hu S, Ma J, Maynard A. Early appraisal of China's huge and complex health-care reforms. Lancet. 2012;379(9818):83342. https://doi.org/10.1016/S0140-6736(11)61880-1.

12. Statistical Bulletin of the Development of Health and Family Planning in China in 2015. http://www.gov.cn/xinwen/2016-07/21/content_5093411.htm.

13. Bonfrer I, van de Poel E, Grimm M, Van Doorslaer E. Does the distribution of healthcare utilization match needs in Africa? Health Policy Plan. 2014;29(7): 921-37. https://doi.org/10.1093/heapol/czt074

14. Núñez A, Chi C. Equity in health care utilization in Chile. Int J Equity Health. 2013;12(1):58. https://doi.org/10.1186/1475-9276-12-58.

15. Flatø $H$, Zhang $H$. Inequity in level of healthcare utilization before and after universal health coverage reforms in China: evidence from household surveys in Sichuan Province. Int J Equity Health. 2016;15(1):96. https://doi. org/10.1186/s12939-016-0385-X

16. Wang $H$, Liu Y, Zhu Y, Xue L, Dale M, Sipsma H, et al. Health insurance benefit design and healthcare utilization in northern rural China. PLoS One. 2012;7(11):e50395. https://doi.org/10.1371/journal.pone.0050395.

17. Kim JH, Lee SG, Lee KS, Jang SI, Cho KH, Park EC. Impact of health insurance status changes on healthcare utilisation patterns: a longitudinal cohort study in South Korea. BMJ Open. 2016;6:e009538. https://doi.org/10.1136/ bmjopen-2015-009538.

18. Tan SY, Wu X, Yang W. Impacts of the type of social health insurance on health service utilisation and expenditures: implications for a unified system in China. Health Econ Policy Law. 2018:1-19. https://doi.org/10.1017/ S174413311800018X.

19. Fontenelle LF, Camargo MBJ, Bertoldi AD, Gonçalves H, Maciel ELN, Barros AJD. Utilization of basic health units of FHS according to private health insurance. Rev Saude Publica. 2018;52:55. https://doi.org/10.11606/S15188787.2018052000383.

20. Chen G, Liu GG, Xu F. The impact of the urban resident basic medical insurance on health services utilisation in China. Pharmacoeconomics. 2014 32(3):277-92. https://doi.org/10.1007/s40273-013-0097-7. 
21. Parmar D, De Allegri M, Savadogo G, Sauerborn R. Do community-based health insurance schemes fulfil the promise of equity? A study from Burkina Faso. Health Policy Plan. 2014;29(1):76-84. https://doi.org/10.1093/heapol/ czs136.

22. Liu X, Wong H, Liu K. Outcome-based health equity across different social health insurance schemes for the elderly in China. BMC Health Serv Res. 2016;16:9. https://doi.org/10.1186/s12913-016-1261-5.

23. Haney M, Shkaratan M. Mine closure and its impact on the community: five years after mine closure in Romania, Russia, and Ukraine (June 12, 2003). World Bank policy research working paper no. 3083. Available at SSRN: https://ssrn.com/abstract=636445.

24. Rosenbaum PR, Rubin DB. The central role of the propensity score in observational studies for causal effects. Biometrika. 1983;70(1):41-55. https:// doi.org/10.1093/biomet/70.1.41.

25. Parsons LS. Reducing bias in a propensity score matched-pair sample using greedy matching techniques. In: proceedings of the twenty-sixth annual SAS users groups international conference, Long Beach, Calif., April 22-25, 2001, Cary, N.C.: SAS Institute, 2001

26. Zhou Z, Zhou Z, Gao J, Yang X, Yan J, Xue Q, et al. The effect of urban basic medical insurance on health service utilisation in Shaanxi Province, China: a comparison of two schemes. PLoS One. 2014;9(4):e94909. https://doi.org/10. 1371/journal.pone.0094909.

27. Kroll LE, Lampert T. Direct costs of inequalities in health care utilization in Germany 1994 to 2009: a top-down projection. BMC Health Serv Res. 2013; 13(1):271. https://doi.org/10.1186/1472-6963-13-271.

28. Zhang C, Lei X, Strauss J, Zhao Y. Health insurance and health care among the mid-aged and older Chinese: evidence from the national baseline survey of CHARLS. Health Econ. 2017;26(4):431-49. https://doi.org/10.1002/ hec.3322.

29. Dou G, Wang $Q$, Ying X. Reducing the medical economic burden of health insurance in. China: Achievements and challenges. Biosci Trends. 2018;12(3): 215-9. https://doi.org/10.5582/bst.2018.01054.

30. Yu J, Qiu Y, He Z. Is universal and uniform health insurance better for China? Evidence from the perspective of supply-induced demand. Health Econ Policy Law. 2018:1-16. https://doi.org/10.1017/S1744133118000385.

\section{Publisher's Note}

Springer Nature remains neutral with regard to jurisdictional claims in published maps and institutional affiliations.

Ready to submit your research? Choose BMC and benefit from:

- fast, convenient online submission

- thorough peer review by experienced researchers in your field

- rapid publication on acceptance

- support for research data, including large and complex data types

- gold Open Access which fosters wider collaboration and increased citations

- maximum visibility for your research: over $100 \mathrm{M}$ website views per year

At $\mathrm{BMC}$, research is always in progress.

Learn more biomedcentral.com/submissions 